\title{
Kaempferol inhibits gastric cancer tumor growth: An in vitro and in vivo study
}

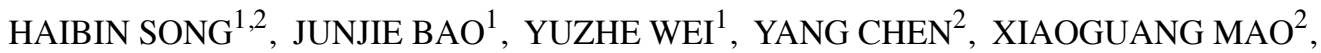 \\ JIANGUO $\mathrm{LI}^{2}$, ZHIWEI YANG ${ }^{2}$ and YINGWEI XUE ${ }^{1}$ \\ ${ }^{1}$ Department of Gastroenterologic Surgery, Harbin Medical University Cancer Hospital, Harbin, Heilongjiang 150040; \\ ${ }^{2}$ Department of General Surgery of Heilongjiang Provincial Hospital, Harbin, Heilongjiang 150036, P.R. China
}

Received August 19, 2014; Accepted November 27, 2014

DOI: $10.3892 /$ or.2014.3662

\begin{abstract}
Kaempferol, which is one of the general flavonoids, has recently been reported to suppress proliferation, induce cell cycle arrest and promote apoptosis in various human cancer cell lines. In the present study, the effect and mechanism of kaempferol on gastric cancer (GC) was examined. The results showed that kaempferol significantly inhibited the proliferation of MKN28 and SGC7901 cell lines. However, no significant inhibition in the GSE-1 normal gastric epithelial cell line in our experimental dose was detected. Additionally, significant apoptosis and G2/M phase cell cycle arrest were identified following the treatment of kaempferol. More importantly, we observed that kaempferol inhibited the growth of the tumor xenografts although no marked effects on liver, spleen or body weight were induced. The expression levels of G2/M cell cycle-regulating factors, cyclin B1, Cdk1 and Cdc25C, were significantly reduced. In addition, kaempferol treatment markedly decreased the level of Bcl-2 concomitant with an increase in Bax expression, resulting in the upregulation of cleaved caspase-3 and -9, which promoted PARP cleavage. Kaempferol-treated cells also led to a decrease in p-Akt, p-ERK and COX-2 expression levels. The present study therefore provided evidence that kaempferol may be a therapeutic agent for GC.
\end{abstract}

\section{Introduction}

On a global scale, gastric cancer (GC) remains the fourth most common malignancy and the second leading cause of cancer-associated mortality (1). Surgical resection remains

Correspondence to: Professor Yingwei Xue, Department of Gastroenterologic Surgery, Harbin Medical University Cancer Hospital, 6 Baojian Road, Harbin, Heilongjiang 150040, P.R. China E-mail: xueyingweijiaoshou@163.com

Abbreviations: GC, gastric cancer; $\mathrm{KF}$, kaempferol; $\mathrm{IC}_{50}$, halfmaximal inhibitory concentration

Key words: gastric cancer, kaempferol, apoptosis, cell cycle arrest the mainstay of curative treatment. However, the majority of patients are diagnosed at an advanced stage when surgery is no longer feasible (2). Consequently, cytotoxic chemotherapy has been proven to be an effective treatment. Nevertheless, drug resistance and therapy-associated side-effects remain issues of concern (3). Thus, more effective antitumor drugs with fewer side-effects for the treatment of GC are required.

A diverse range of phytochemicals have confirmed the capacity to selectively inhibit the growth of tumor cells (4-8). Flavonoids are polyphenolic compounds that have a wide range of biological activities, including antioxidant ability, anti-inflammatory capacity and ability to combat cancer $(9,10)$. Kaempferol is one of the general flavonoids that is often present in tea, broccoli, apples, strawberries and beans (11). It has received much attention due to its anticancer potential, preferable biocompatibility and few side-effects. Kaempferol has been found to inhibit proliferation, angiogenesis and metastasis, induce cell cycle arrest and promote apoptosis in a variety of human cancer cell lines (12-19). However, the potential role of these phytochemicals in GC treatment has yet to be evaluated. Therefore, we investigated the therapeutic potential and molecular mechanisms of kaempferol on GC cells in vitro and in vivo.

Results of the present study demonstrated that kaempferol potently inhibits the proliferation of GC cells and induces apoptosis via the mitochondrial pathway in vitro and in vivo. $\mathrm{G} 2 / \mathrm{M}$ arrest of GC cells was also observed and the expression levels of $\mathrm{G} 2 / \mathrm{M}$ cell cycle regulating factors, cyclin B1, Cdk1 and $\mathrm{Cdc} 25 \mathrm{C}$, were decreased following kaempferol treatment. Additionally, we observed that kaempferol inhibited the ERK1/2 and PI3K/AKT signaling pathway. In conclusion, the present study results revealed the therapeutic potential of kaempferol in GC, and we demonstrated several possible mechanisms that may be significant in the activity of kaempferol, although this remains to be determined in future studies.

\section{Materials and methods}

Cell lines, reagents and antibodies. MKN28, SGC7901 and GSE-1 cells were purchased from the Cell Resource Center of Shanghai Institutes for Biological Sciences, Chinese Academy of Sciences (Shanghai, China). All the cells were cultured in RPMI-1640 medium (Gibco-BRL) supplemented with 10\% 
fetal bovine serum (FBS) at $37^{\circ} \mathrm{C}$ and $5 \% \mathrm{CO}_{2}$. Kaempferol was purchased from Winherb Medical S\&T Development Co., Ltd. (Shanghai, China). Primary antibodies against Bcl-2, Bax, survivin, Bcl-xL, caspase-3, cleaved-caspase-3, caspase-9, cleaved-caspase-9, PARP, cleaved-PARP, p-Akt, Akt, cyclin B1, Cdk1, Cdc25C, GAPDH and secondary antibodies against mouse IgG-horseradish peroxidase (HRP) and rabbit IgG-HRP were obtained from Santa Cruz Biotechnology, Inc. (Santa Cruz, CA, USA). Antibodies against ERK1/2, phosphoERK1/2, Ki67 and COX-2 were purchased from Cell Signaling Technology, Inc. (Trask Lane, Danvers, MA, USA).

Cell viability assay. To investigate cell viability MKN28, SGC7901 and GSE-1 cells were seeded in 96-well plates at a density of $3 \times 10^{3} /$ well and cultured for $24 \mathrm{~h}$. After validation of cell adherence, the cells were treated with different doses of kaempferol for 24, 48 or $72 \mathrm{~h}$. A Cell Counting Kit-8 (CCK-8; Dojindo Molecular Technologies, Kumamoto, Japan) was used to assess the cell viability. Cell viability was calculated as a percentage of absorbance in treated wells relative to that of untreated wells. Three independent experiments were performed.

Cell cycle analysis. Cells were seeded in 6-well plates at $4 \times 10^{5} /$ dish. After incubation with kaempferol (60 or $\left.120 \mu \mathrm{M}\right)$ for $48 \mathrm{~h}$, the cells were trypsinized and washed twice with phosphate-buffered saline (PBS). The cells were then cultured with reagents A-C according to the manufacturer's instructions and subjected to flow cytometry.

Analysis of apoptosis. GC cells $\left(4 \times 10^{5}\right.$ cells/well) were incubated with kaempferol $(60$ or $120 \mu \mathrm{M})$ for $48 \mathrm{~h}$. Cells $\left(1 \times 10^{4}\right)$ were collected and washed twice with cold PBS. Apoptotic cells were evaluated by double staining with propidium iodide (PI) and Annexin V labeled with FITC using an Annexin V-FITC apoptosis detection kit (BD Biosciences, San Jose, CA, USA) according to the manufacturer's instructions.

Western blotting. MKN28 and SGC7901 cells were plated at a density of $4 \times 10^{5} /$ well in 6 -well plates. After incubation with kaempferol $(60$ or $120 \mu \mathrm{M})$ the cells were washed twice with ice-cold PBS and treated with $120 \mu 1$ sample buffer on ice for $30 \mathrm{~min}$. The cell lysate was centrifuged at $12,000 \mathrm{rpm}$ for $10 \mathrm{~min}$ at $4^{\circ} \mathrm{C}$. Protein lysates $(20 \mu \mathrm{l})$ were electrophoresed on a 12 or $10 \%$ SDS gel. The proteins were then electrotransferred to a PVDF membrane and the membrane was blocked for 30 min with blocking solution (5\% non-fat dry milk in PBS-0.5\% Tween-20). The membrane was then incubated overnight at $4^{\circ} \mathrm{C}$ with primary antibodies $(1: 1,000)$. Subsequently, the membrane was washed in PBST for $30 \mathrm{~min}$, exposed to HRP-conjugated secondary antibody (diluted 1:2,000), and washed again in PBST for $30 \mathrm{~min}$. Final detection was performed using enhanced chemiluminescence solution for $5 \mathrm{~min}$.

Ki-67 immunohistochemistry. Formalin-fixed, paraffinembedded sections $(5 \mathrm{~mm}$ ) were rinsed with PBS, blocked with $10 \%$ bovine serum albumin for $30 \mathrm{~min}$ and then stained with an anti-Ki-67 antibody overnight. The sections were subsequently incubated for $1 \mathrm{~h}$ with the appropriate secondary antibody,
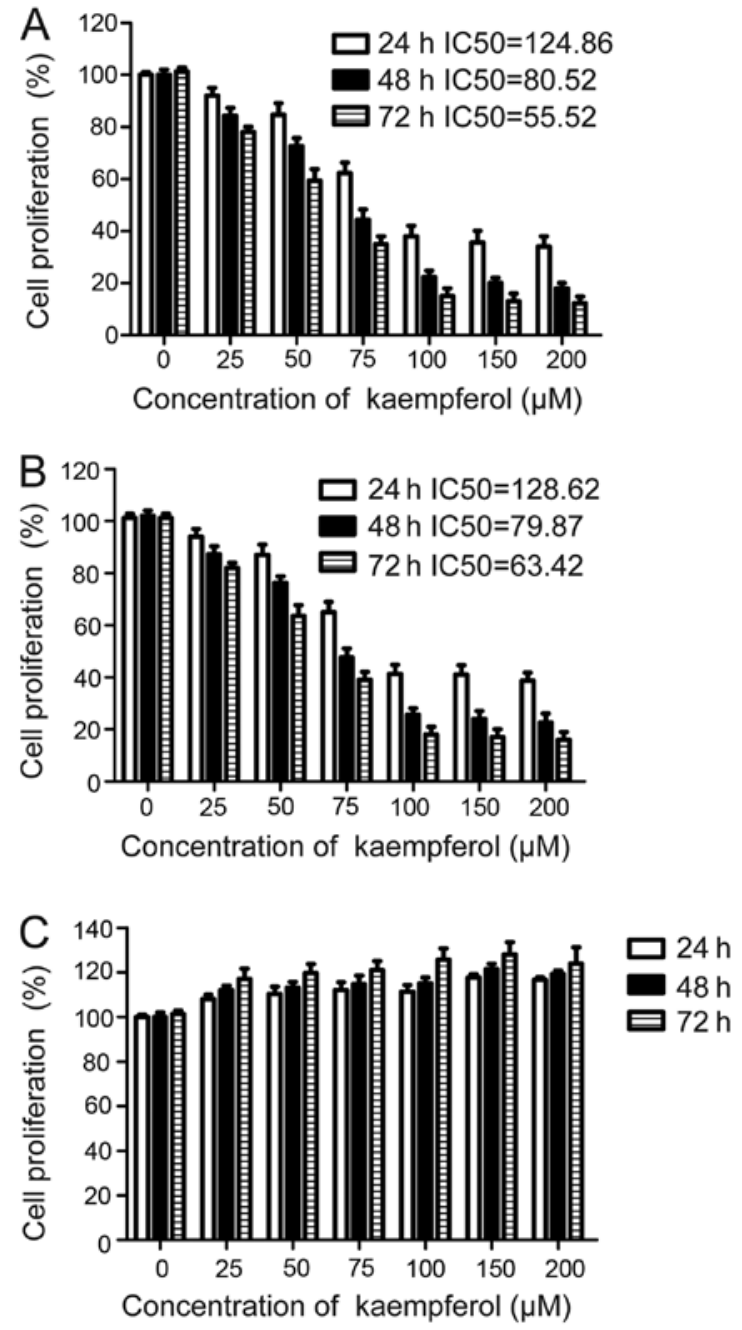

Figure 1. Kaempferol treatment on proliferation of MKN28, SGC7901 and GSE-1 cells. (A-C) Cells were incubated with increasing doses of kaempferol (25-200 $\mu \mathrm{M}$ ) for 24-72 $\mathrm{h}$ periods and analyzed for cell proliferation using Cell Counting Kit-8 assay.

and immunoreactivity was developed with SigmaFAST DAB. Positively stained cells from three tumors/group were counted in 10 randomly selected fields under $x 400$ high-power magnification. A proliferative index (\%) was calculated using the formula: Number of Ki-67-positive cells/total cell count.

Tumor xenograft experiments. SGC7901 cells $\left(3 \times 10^{6}\right)$ were injected into the flanks of 4-week-old athymic mice to establish the tumor. When the tumor volume reached $100 \mathrm{~mm}^{3}$, kaempferol $(20 \mathrm{mg} / \mathrm{kg})$ was administered i.p. for 3 weeks daily. The mice in the treatment and control groups $(n=8$ in each group) were sacrificed to obtain the snap-frozen and paraffin-embedded tumor tissue for further analysis. Body weight was recorded starting from the first day of treatment, and tumor volumes were also calculated at the same time points using the equation: Tumor volume $=$ length $\mathrm{x}$ (width) $)^{2} \mathrm{x} \pi / 6$. Operative procedures and care were approved by the Institutional Ethics Committee at Harbin Medical University. All the experiments were performed in accordance with the guidelines of the Committee on the Use of Live Animals in Teaching and Research of Harbin Medical University, Harbin, China. 

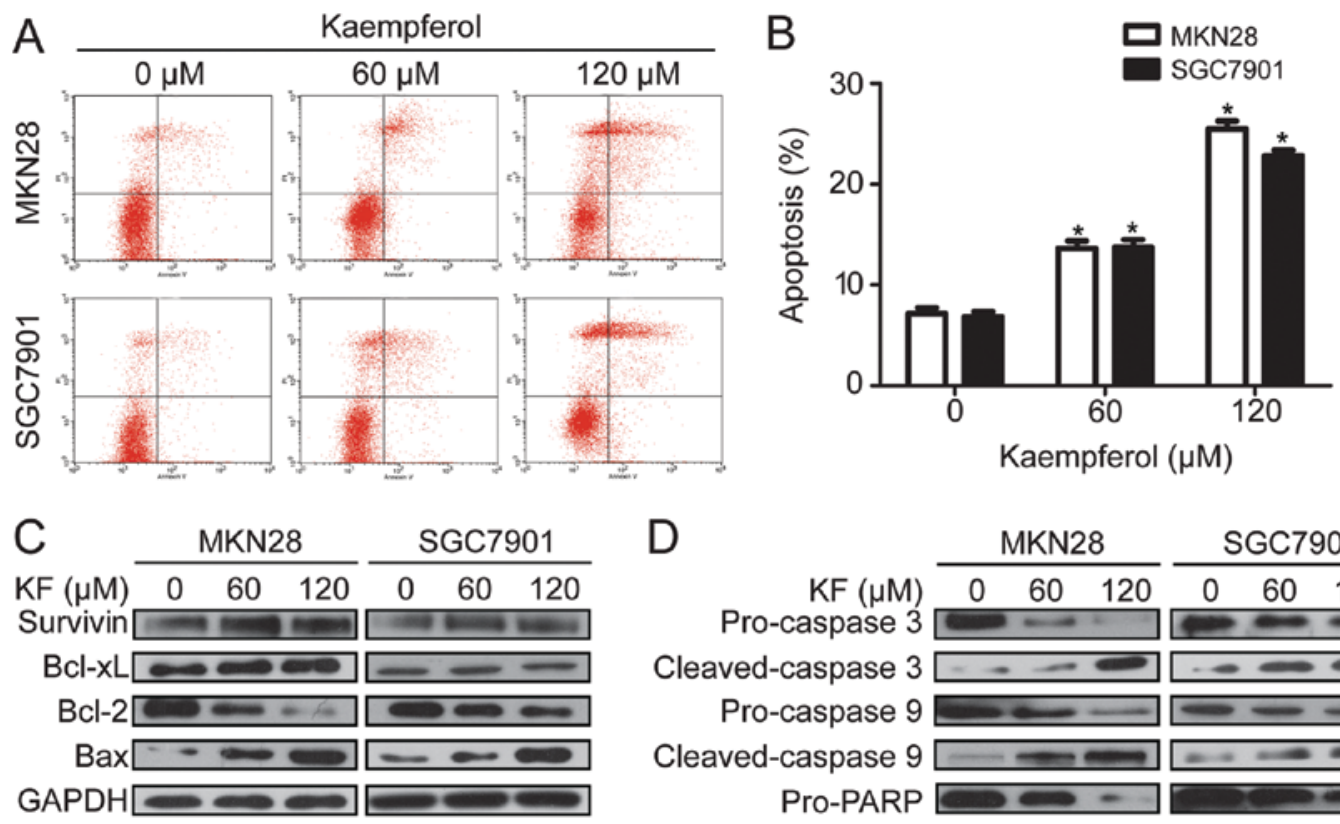

D
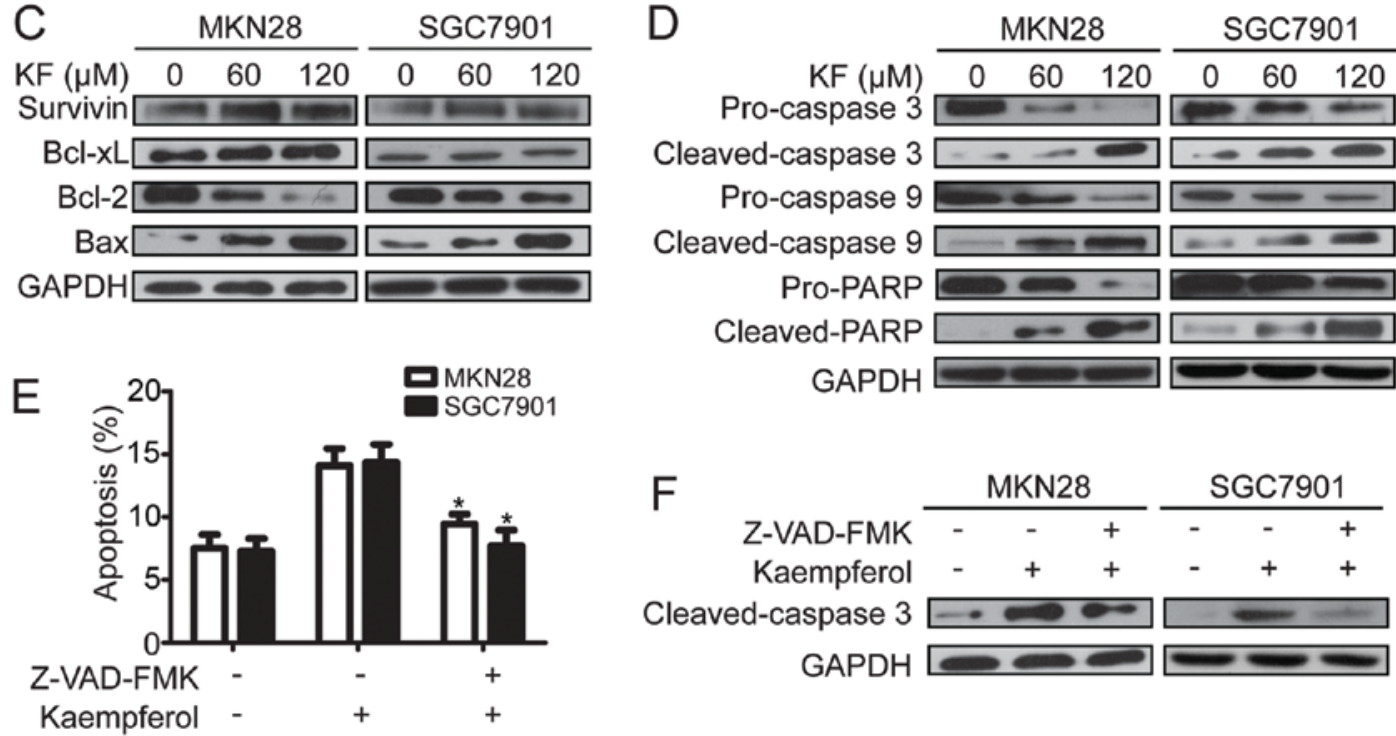

Figure 2. Kaempferol induces gastric cancer cell apoptosis. (A) MKN28 and SGC7901 cells incubated with 60 or $120 \mu$ M kaempferol for $48 \mathrm{~h}$ were analyzed for apoptosis. (B) Representative histograms from cytometrically analyzed the two cell lines treated with control and kaempferol. Assay was carried out in triplicate. ${ }^{*} \mathrm{P}<0.05$. (C and D) Apoptosis-associated proteins were detected by western blotting. (E and F) Changes in cleaved-caspase 3 and histograms of the apoptotic rate from the cell lines treated with kaempferol, and kaempferol in combination with pan-caspase inhibitor (Z-VAD-fmk).

Statistical analysis. Data were presented as mean values \pm standard deviation (SD). Analysis of variance (ANOVA) and a Student's t-test were used to evaluate statistical significance. $\mathrm{P}<0.05$ was considered to indicate a statistically significant result.

\section{Results}

Kaempferol inhibits GC cell proliferation. First, we assessed the effects of kaempferol on the cell proliferation of GC cell lines. We used MKN28 and SGC7901 GC cell lines, which are widely utilized in GC investigations to examine the effects of kaempferol. We also used a GSE-1 cell line to determine whether kaempferol had the same effects on normal gastric epithelial cells. The results showed that kaempferol significantly inhibited the proliferation of all the GC cell lines within a period of 24-72 $\mathrm{h}(\mathrm{P}<0.05)$, and these effects were more apparent at a dose of $120 \mu \mathrm{M}$ (Fig. 1A and B). However, no marked inhibition was observed in the GSE-1 cell line with our experimental dose (Fig. 1C).

Kaempferol induces apoptosis in GC cells. To determine whether kaempferol suppressed cell proliferation by inducing apoptosis, we used an Annexin V/PI assay as described above to investigate the apoptotic effect on GC cells. The results showed that kaempferol induced the apoptosis of SGC-7901 and MKN28 cells in a dose-dependent manner (Fig. 2A and B). When the concentration of kaempferol reached $60 \mu \mathrm{M}$, the apoptotic rate of the GC cell lines was markedly higher than that of the untreated cells $(\mathrm{P}<0.05)$. Moreover, $120 \mu \mathrm{M}$ kaempferol resulted in a higly significant difference in the rate of apoptosis compared to the kaempferol-treated $(60 \mu \mathrm{M})$ and untreated cells $(\mathrm{P}<0.05)$. As apoptosis is regulated by a variety of pro- and anti-apoptotic proteins (20-22), we investigated the expression levels of apoptosis-associated proteins, including $\mathrm{Bcl}-2, \mathrm{Bax}, \mathrm{Bcl}-\mathrm{xL}$ and survivin. The results showed that kaempferol decreased the expression of $\mathrm{Bcl}-2$ and concomitantly increased the expression of Bax in a concentration-dependent manner (Fig. 2C). However, no significant change of Bcl-xL and survivin was observed in the GC cell line. Caspase family proteins are important enzymes to execute apoptosis.

We also assessed the role of kaempferol in the caspase cascade pathway. Western blotting showed that kaempferol treatment lead to a dose-dependent elevation of cleaved caspase- 3 and -9 , and cleaved-PARP, and a dose-dependent decrease of pro-caspase-3 and -9, and pro-PARP (Fig. 2D). To 

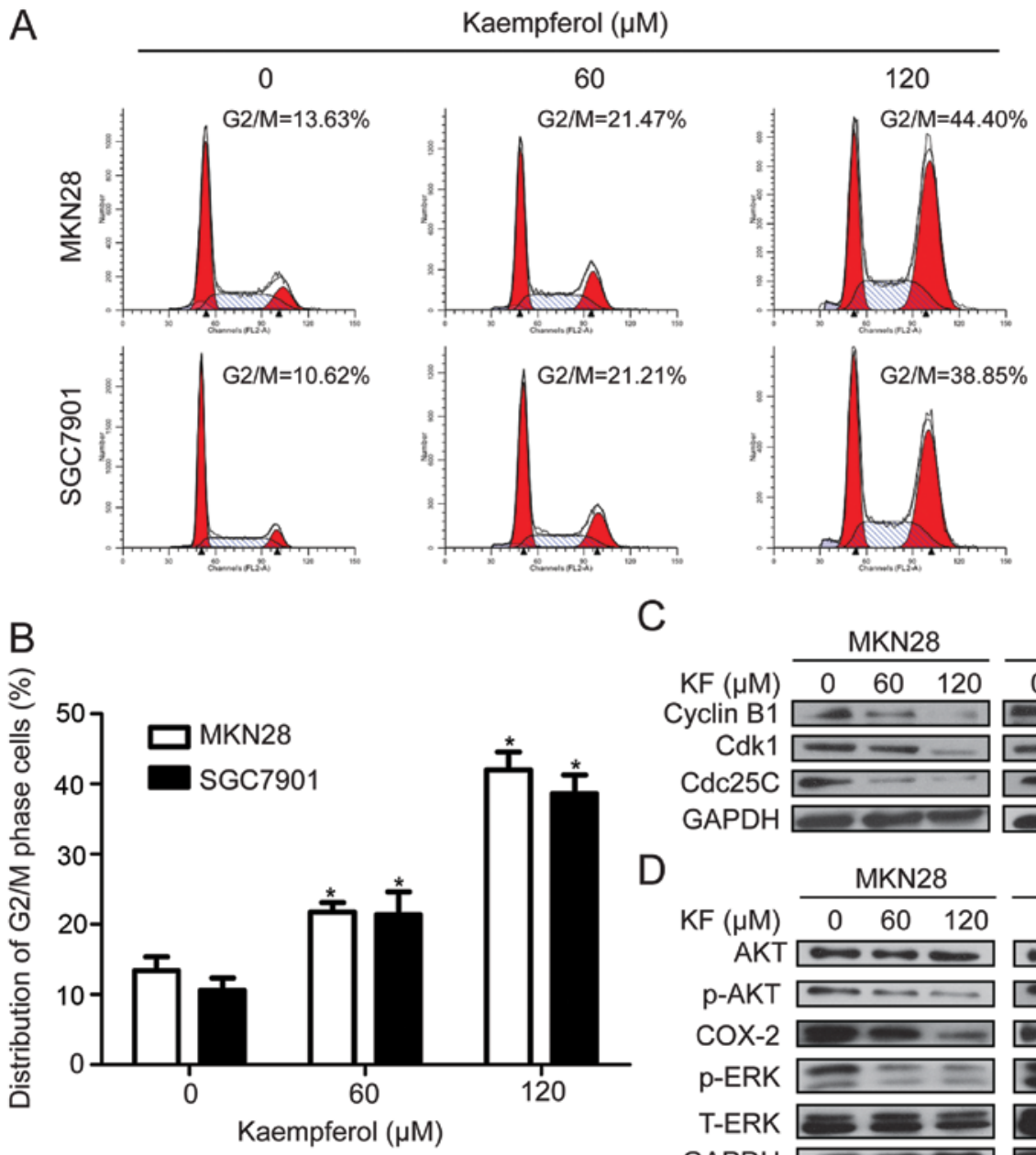

C

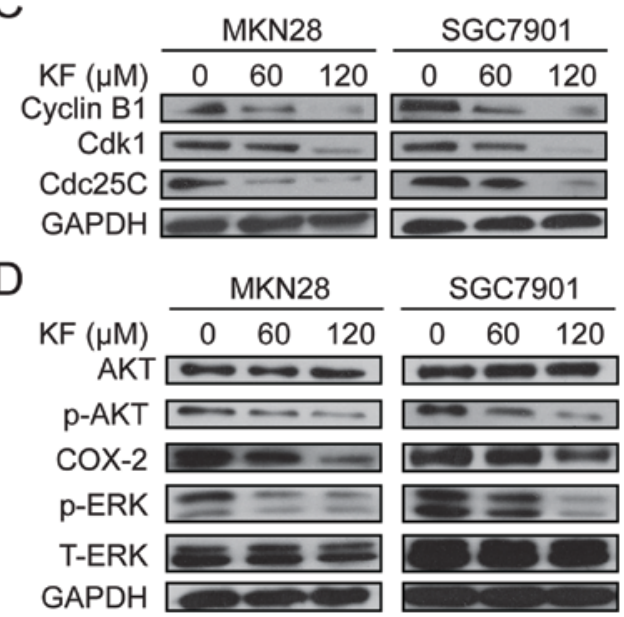

Figure 3. Kaempferol induces cell cycle arrest and inhibits tumor cell survival signaling in gastric cancer cells. Gastric cancer cells were treated with DMSO (control) or different concentrations of kaempferol for $48 \mathrm{~h}$ for flow cytometric analysis and western blotting. (A) Representative flow cytometry graph for each treated or untreated groups. (B) Representative histogram for the two cytometrically analyzed cell lines treated with DMSO (control) or kaempferol. Assay was carried out in triplicate. ${ }^{*} \mathrm{P}<0.05$. (C) Expression of G2/M cell cycle-associated proteins, cyclin B1, Cdk1 and Cdc25C were evaluated by western blotting following treatment with 60 or $120 \mu \mathrm{M}$ kaempferol for $48 \mathrm{~h}$. (D) Western blotting was used to analyze the expressions of ERK, p-ERK, Akt, p-Akt and COX-2.

further investigate the role of caspase activation in kaempferolinduced apoptosis, we treated SGC-7901 and MKN28 cells with pan-caspase inhibitor Z-VAD-FMK (10 mmol/l) before kaempferol treatment. The pan-caspase inhibitor Z-VAD-FMK pretreatment reduced the expression of cleaved caspase-3 and kaempferol-induced apoptosis (Fig. 2E and F). These data demonstrated that kaempferol induced the apoptosis of GC cells by regulating the expression levels of apoptosis-related proteins and partly activating caspase-dependent cell death pathway.

Kaempferol induces $G 2 / M$ cell cycle arrest and inhibits tumor cell survival signaling in GC cells. To examine the underlying mechanism of reduced cell viability observed in the CCK-8 assay, we examined the cell distribution by flow cytometry following treatment with 60 or $120 \mu \mathrm{M}$ kaempferol for $48 \mathrm{~h}$. Kaempferol treatment for $48 \mathrm{~h}$ arrested cells at the G2/M stage (Fig. 3A and B). To investigate the mechanism for $\mathrm{G} 2 / \mathrm{M}$ arrest in kaempferol-treated cells, we detected the expression of proteins that are pivotal for $\mathrm{G} 2 / \mathrm{M}$ transition, including cyclin B1, Cdk1 and Cdc25C. The results showed that kaempferol led to a marked decrease in the protein levels of cyclin B1, Cdk1 and $\mathrm{Cdc} 25 \mathrm{C}$ in a dose-dependent manner (Fig. 3C). These data revealed that the inhibitory effect of kaempferol on GC was associated with the induction of $\mathrm{G} 2 / \mathrm{M}$ phase arrest. Our data indicated that in vitro kaempferol treatment significantly reduced the expression levels of COX-2, p-AKT and p-ERK, which were involved in cell proliferation and cell cycle arrest. However, kaempferol had no impact on the level of total Akt and ERK (Fig. 3D). These findings revealed that kaempferol suppresses tumor cell growth via the PI3K/AKT or ERK-MAPK pathway.

Kaempferol suppresses tumor growth in vivo. We established a subcutaneous tumor model, which was derived from the SGC7901 cell line, to examine the role of kaempferol in tumor growth in vivo. SGC7901 cell-derived xenograft tumors were allowed to develop and grow to a size of $100 \mathrm{~mm}^{3}$, and then kaempferol (20 mg/kg/day) was administered i.p. for 3 weeks daily. The mice were sacrificed following 3 weeks of kaempferol treatment and tumors were excised. Data showed that kaempferol significantly suppressed the growth of the tumor xenografts (Fig. 4A). However, no marked change was observed in body, liver or spleen weight in the animals (Fig. 4B). The 
A

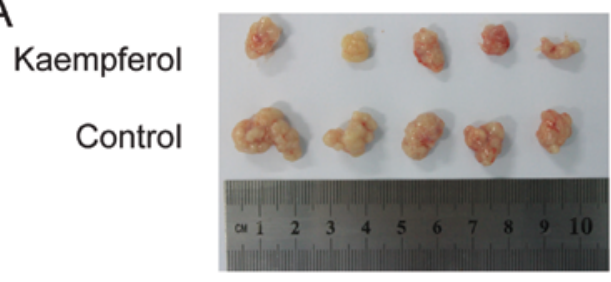

B
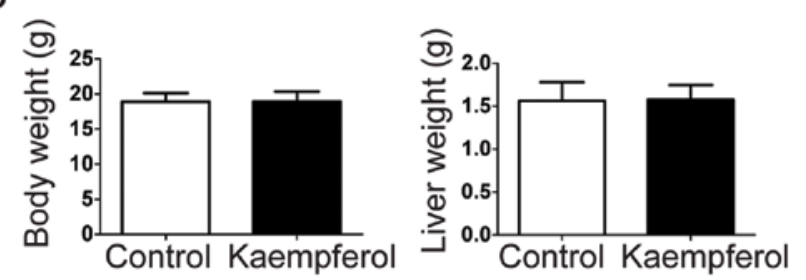
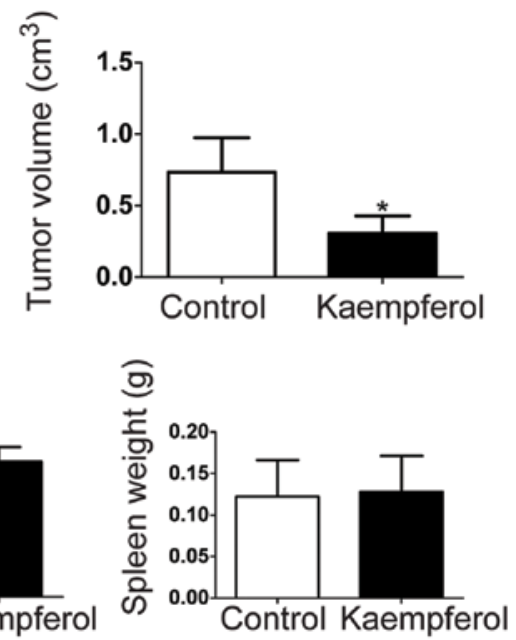

\section{C}
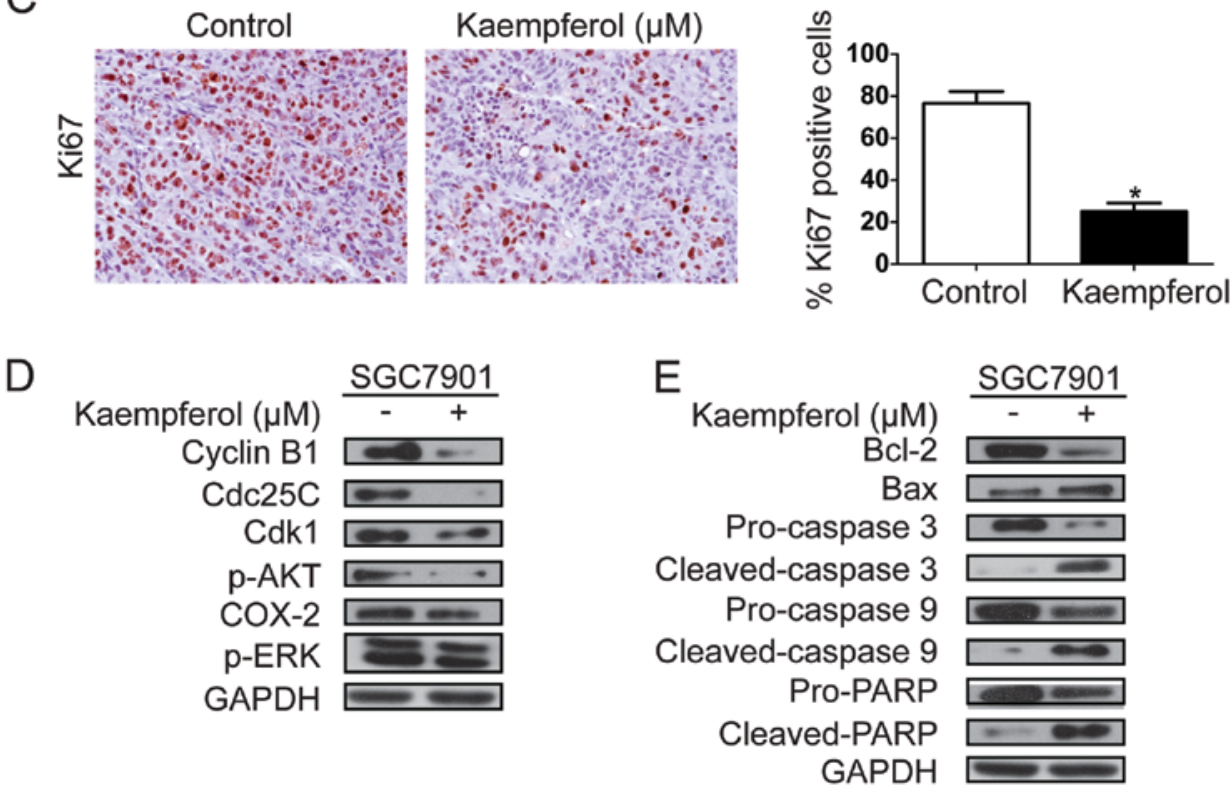

Figure 4. Kaempferol suppresses tumor growth in vivo. (A and B) SGC7901 cells were injected into the flanks of nude mice, and xenograft tumors were allowed to develop and grow to a size of $100 \mathrm{~mm}^{3}$, and then kaempferol $(20 \mathrm{mg} / \mathrm{kg})$ was administered i.p. for 3 weeks daily. On day 22 , the tumors were excised and subjected to further analyses. The tumor volumes of kaempferol-treated mice were smaller than those of the control mice. ${ }^{*}<0.05$. (C) There was no significant change in body, liver and spleen weight between control and kaempferol-treated animals. (D and E) Western blot analysis on the expression of apoptosis-associated proteins, cell cycle regulated proteins and proteins of survival signaling.

results revealed that kaempferol was a potential therapeutic drug for the treatment of GC and it was relatively non-toxic to mice. Ki-67 staining for cell proliferation was performed in these xenografts, and the number of $\mathrm{Ki}$-67-positive tumor cells was lower in tumors from kaempferol-treated mice than that from non-kaempferol-treated mice (Fig. 4C). Western blot analysis revealed that kaempferol treatment decreased the expression of cyclin B1, Cdk1, Cdc25C, Bcl-2/Bax ratio, p-AKT, P-ERK and increased the expression of cleaved caspase- 3 and -9 , and cleaved PARP (Fig. 4D and E). These findings demonstrated that kaempferol significantly suppressed GC cell growth in vivo.

\section{Discussion}

Despite recent advancement in revealing the tumorigenetic mechanism of gastric cancer (GC), the ever expanding incidence and relatively low relief rate of chemotherapy have led to identification of a more efficacious treatment method. The present study shows that kaempferol significantly suppressed the proliferation of GC cells. Moreover, it did not influence the proliferation of normal gastric GSE-1 cells when treated with a concentration of $120 \mu \mathrm{M}$.

Bcl-2 family proteins, including pro-apoptotic proteins (Bid, Bak and Bax) and anti-apoptotic proteins (Bcl-2, cIAP-2, XIAP, Bcl-xL and survivin), are critical in the control of apoptosis $(23,24)$. The ratio of anti- and pro-apoptotic protein expression, such as Bcl-2/Bax, is crucial for the induction of apoptosis, and it determines the susceptibility of cells to undergo apoptosis (25). However, Bcl-2 family proteins conduct the release of cytochrome $c$ from the mitochondria into cytosol, which lead to the activation of caspase- 9 and induces a subsequent caspase cascade (the intrinsic cell death pathway). Luo et al showed that kaempferol induced apoptosis in ovarian cancer cells through the intrinsic apoptosis pathway (16). In 
the present study, we observed that kaempferol increased the expression of Bax and decreased the expression of Bcl-2 in SGC-7901 and MKN28 cells. Moreover, the result of kaempferol-induced activation of caspase-3 and -9 , and subsequent cleavage of PARP, as well as the data that the pan-caspase inhibitor Z-VAD-FMK decreased kaempferol-mediated apoptosis in SGC-7901 and MKN28 cells, indicate that kaempferol induced the apoptosis of GC cells partly through a mitochondrial cell death pathway.

Eukaryotic cell cycle progression is involved in the successive activation of Cdks whose activation is dependent on their conjunction with cyclins. A complex shaped by the conjunction of $\mathrm{Cdk} 1$ and cyclin B1 plays an important role at entry into mitosis (26). Choi and Ahn showed that kaempferol induced G2/M phase cell cycle arrest in MDA-MB-453 human breast cancer cells (13). The results of the present study showed that the treatment of MKN28 and SGC7901 cells with kaempferol led to the arrest of GC cells in G2/M phase and that kaempferol-mediated G2/M arrest is connected with a decrease in the protein levels of $\mathrm{Cdk} 1$, cyclin B1 and Cdc25C. Therefore, kaempferol may induce cell cycle arrest by decreasing activity of the Cdk1/cyclin B kinase complex through downregulation of various $\mathrm{G} 2 / \mathrm{M}$-associated proteins.

The PI3K-Akt signaling pathway is a crucial regulator of a number of cell processes including proliferation, differentiation and metastasis in cancer development (27). Activated AKT phosphorylates numerous proteins that have been involved in the control of the cell cycle to ultimately lead to cell growth and suppress apoptosis (28). As kaempferol can block the $\mathrm{PI} 3 \mathrm{~K} / \mathrm{AKT}$ pathway in mouse epidermal $\mathrm{JB}^{+} \mathrm{P}^{+}$cells by neutralizing PI3K (29), we determined whether the effect of kaempferol on GC cells was associated with the inhibition of this pathway as well. Our data showed that the expression level of p-Akt was reduced in a dose-dependent manner, without any changes in the total Akt protein level following kaempferol treatment. ERK is known to be involved in the promotion of cell proliferation and is generally upregulated in many cancers, including GC. Results of this study also showed a marked dosedependent reduced ERK phosphorylation in cells treated with kaempferol. Therefore, we suggest that kaempferol suppresses GC through the PI3K/AKT and ERK-MAPK pathway, highlighting a potential mechanism for kaempferol activity, which may be used as a therapeutic agent for GC. Nevertheless, the exact mechanism should be further investigated.

The in vivo antitumor effect of kaempferol was analyzed in a SGC7901 xenograft tumor model in nude mice. An apparent decrease in the relative tumor volume was observed in kaempferol-treated mice compared to non-kaempferol-treated controls. However, a significant inhibition of proliferation was observed on the results of immunohistochemistry for Ki-67 in kaempferol-treated mice. Of note, kaempferol treatment did not affect liver, spleen or total body weight. Although kaempferol seemed to have potent antitumor activity with few side effects in the present study, absorption and pharmacokinetic properties of kaempferol need to be further investigated to confirm kaempferol as an efficacious therapy for GC.

Kaempferol, which is a natural flavonoid present in various fruits and vegetables, exerts antitumor activity in a variety of cancer cells. The antitumor role of kaempferol and its underlying mechanisms was assessed in the MKN28 and
SGC7901 GC cell lines. Kaempferol was found to suppress the proliferation of the two GC cell lines, however, no significant inhibition effect was observed in normal gastric epithelial cell line, GSE-1. G2/M phase cell cycle arrest and apoptosis were observed by using flow cytometry. Accordingly, kaempferol treatment downregulated the expression of G2/M cell cycle-associated proteins cyclin B1, Cdk1 and Cdc25C. Kaempferol treatment also upregulated the expression of Bax concomitant with a decrease in Bcl-2 and increased the expression of cleaved caspase- 3 and -9 , and cleaved PARP. We also observed that kaempferol decreased the protein level of p-Akt and p-ERK in MKN28 and SGC7901 cells. In vivo kaempferol significantly suppressed the growth of the tumor xenografts with no marked change in liver, spleen or body weight and protein expression data in vitro were further confirmed. In conclusion, our data provide a basis for further inquiry of kaempferol as a therapeutic agent for GC.

\section{Acknowledgements}

We would like to thank Dr Y.H. Gu for his support in the pathobiology examination. This study was supported by Foundation of Health and Family Planning Commission of Heilongjiang Province.

\section{References}

1. Alberts SR, Cervantes A and van de Velde CJ: Gastric cancer: epidemiology, pathology and treatment. Ann Oncol 14 (Suppl 2): ii31-ii36, 2003.

2. Hundahl SA, Menck HR, Mansour EG and Winchester DP: The National Cancer Data Base report on gastric carcinoma. Cancer 80: 2333-2341, 1997.

3. Ajani JA: Evolving chemotherapy for advanced gastric cancer. Oncologist 10 (Suppl 3): S49-S58, 2005.

4. Aggarwal BB and Shishodia S: Molecular targets of dietary agents for prevention and therapy of cancer. Biochem Pharmacol 71: 1397-1421, 2006.

5. Naithani R, Huma LC, Moriarty RM, McCormick DL and Mehta G: Comprehensive review of cancer chemopreventive agents evaluated in experimental carcinogenesis models and clinical trials. Curr Med Chem 15: 1044-1071, 2008.

6. Kaefer CM and Milner JA: The role of herbs and spices in cancer prevention. J Nutr Biochem 19: 347-361, 2008.

7. Russo GL: Ins and outs of dietary phytochemicals in cancer chemoprevention. Biochem Pharmacol 74: 533-544, 2007.

8. Moiseeva EP and Manson MM: Dietary chemopreventive phytochemicals: too little or too much? Cancer Prev Res 2: 611-616, 2009.

9. Seifried HE, Anderson DE, Fisher EI and Milner JA: A review of the interaction among dietary antioxidants and reactive oxygen species. J Nutr Biochem 18: 567-579, 2007.

10. Luo H, Jiang BH, King SM and Chen YC: Inhibition of cell growth and VEGF expression in ovarian cancer cells by flavonoids. Nutr Cancer 60: 800-809, 2008.

11. Somerset SM and Johannot L: Dietary flavonoid sources in Australian adults. Nutr Cancer 60: 442-449, 2008.

12. Zhang Y, Chen AY, Li M, Chen C and Yao Q: Ginkgo biloba extract kaempferol inhibits cell proliferation and induces apoptosis in pancreatic cancer cells. J Surg Res 148: 17-23, 2008.

13. Choi EJ and Ahn WS: Kaempferol induced the apoptosis via cell cycle arrest in human breast cancer MDA-MB-453 cells. Nutr Res Pract 2: 322-325, 2008.

14. Luo H, Rankin GO, Liu L, Daddysman MK, Jiang BH and Chen YC: Kaempferol inhibits angiogenesis and VEGF expression through both HIF dependent and independent pathways in human ovarian cancer cells. Nutr Cancer 61: 554-563, 2009.

15. Luo H, Daddysman MK, Rankin GO, Jiang BH and Chen YC: Kaempferol enhances cisplatin's effect on ovarian cancer cells through promoting apoptosis caused by down regulation of cMyc. Cancer Cell Int 10: 16, 2010. 
16. Luo H, Rankin GO, Li Z, Depriest L and Chen YC: Kaempferol induces apoptosis in ovarian cancer cells through activating p53 in the intrinsic pathway. Food Chem 128: 513-519, 2011.

17. Nguyen TT, Tran E, Ong CK, et al: Kaempferol-induced growth inhibition and apoptosis in A549 lung cancer cells is mediated by activation of MEK-MAPK. J Cell Physiol 197: 110-121, 2003

18. Chen HJ, Lin CM, Lee CY, et al: Kaempferol suppresses cell metastasis via inhibition of the ERK-p38-JNK and AP-1 signaling pathways in U-2 OS human osteosarcoma cells. Oncol Rep 30: 925-932, 2013.

19. Song W, Dang Q, Xu D, et al: Kaempferol induces cell cycle arrest and apoptosis in renal cell carcinoma through EGFR/p38 signaling. Oncol Rep 31: 1350-1356, 2014.

20. Debatin KM: Apoptosis pathways in cancer and cancer therapy. Cancer Immunol Immunother 53: 153-159, 2004

21. Fisher DE: Pathways of apoptosis and the modulation of cell death in cancer. Hematol Oncol Clin North Am 15: 931-956, ix, 2001.

22. Fulda $S$ and Debatin KM: Targeting apoptosis pathways in cancer therapy. Curr Cancer Drug Targets 4: 569-576, 2004.
23. Susnow N, Zeng L, Margineantu D and Hockenbery DM: Bcl-2 family proteins as regulators of oxidative stress. Semin Cancer Biol 19: 42-49, 2009

24. Daniel PT, Schulze-Osthoff K, Belka C and Güner D: Guardians of cell death: the Bcl-2 family proteins. Essays Biochem 39: 73-88, 2003.

25. Cory S and Adams JM: The Bcl 2 family: regulators of the cellular life-or-death switch. Nat Rev Cancer 2: 647-656, 2002.

26. Molinari M: Cell cycle checkpoints and their inactivation in human cancer. Cell Prolif 33: 261-274, 2000.

27. Cully M, You H, Levine AJ and Mak TW: Beyond PTEN mutations: the PI3K pathway as an integrator of multiple inputs during tumorigenesis. Nat Rev Cancer 6: 184-192, 2006.

28. Chang F, Lee JT, Navolanic PM, et al: Involvement of PI3K/Akt pathway in cell cycle progression, apoptosis, and neoplastic transformation: a target for cancer chemotherapy. Leukemia 17: 590-603, 2003.

29. Lee KM, Lee DE, Seo SK, et al: Phosphatidylinositol 3-kinase, a novel target molecule for the inhibitory effects of kaempferol on neoplastic cell transformation. Carcinogenesis 31: 1338-1343, 2010 . 